\title{
Beam propagation method analysis of transverse-electric waves propagating in a nonlinear tapered planar waveguide
}

\author{
Tian-Tsorng Shi and Sien Chi \\ Institute of Electro-Optical Engineering and Center for Telecommunications Research, \\ National Chiao Tung University, Hsinchu, Taiwan, China
}

Received October 22, 1990; revised manuscript received June 26, 1991

\begin{abstract}
The propagation of TE-polarized guided waves in a nonlinear tapered waveguide is studied. The incident fields are chosen from the stable branches of the nonlinear dispersion curve in its uniform section. The propagations of the field in both directions are calculated by the beam-propagation method. Wave propagation in the tapered waveguide is discussed in terms of the nonlinear dispersion curves of the waveguide. Depending on the intensity, the field can radiate as a spatial soliton or it can have an abrupt transition between the guided mode and the surface polariton. The wave propagation of an incident field that is composed of a dominant $\mathrm{TE}_{0}$ wave and a small $\mathrm{TE}_{1}$ wave is investigated. Finally, using a property of this wave propagation, we present a new device for continuous signal routing.
\end{abstract}

\section{INTRODUCTION}

Wave propagation in nonlinear waveguides has been studied extensively, ${ }^{1-5}$ and many nonlinear integrated optical devices have been suggested. ${ }^{6-15}$ Among them, the nonlinear directional coupler has received the most attention, and its coupling characteristics depend on its input light intensity. The nonlinear $\mathrm{X}$ junction is one of the most promising devices for future applications; in it a strong signal beam can be routed by a weaker control beam.

However, wave propagation in the nonlinear tapered waveguide has been less extensively discussed. ${ }^{16}$ In a linear multimode waveguide that is tapered to a single-mode waveguide the higher-order eigenmodes radiate out, and only the fundamental eigenmode remains in the singlemode waveguide. In a nonlinear waveguide the shape of the field and the propagation constant of the eigenmode depend on the intensity of the field. The nonlinear dispersion relations have been calculated for several different structures. ${ }^{17,18}$ We use the nonlinear dispersion curves of the nonlinear tapered waveguide to explain the wave propagation in it. The waveguide consists of a linear film that is bounded by a linear substrate and a nonlinear self-focus cladding. The field propagating in this nonlinear tapered waveguide varies as the power of the incident field. In this paper only TE waves are considered. TM wave propagation can be calculated by the finite-element method. ${ }^{16}$

Wave propagation will be simulated by the beam propagation method, ${ }^{19}$ and its behavior will be explained by the nonlinear dispersion curves of the waveguide. The propagation of the fundamental mode is considered first. Then the propagation of the incident wave, composed of a dominant $\mathrm{TE}_{0}$ wave and a small $\mathrm{TE}_{1}$ wave, is investigated. Finally, a new optical switching or scanning device is proposed.

In Section 2 we calculate the nonlinear dispersion curves of the waveguide for several specific film thicknesses. In Section 3 we present some numerical results for wave propagation in the tapered waveguide. A pro- posal for optical switching and scanning is presented in Section 4. Section 5 concludes this paper.

\section{NONLINEAR DISPERSION RELATIONS}

Here we briefly summarize the formulas that describe the fields and nonlinear dispersion relations. The TE wave, polarized in the $y$ direction and propagating in the planar waveguide, is written as

$$
E_{y}(x, z, t)=1 / 2 E_{y}(x) \exp \left[i\left(k_{0} \beta z-\omega t\right)\right]+\text { c.c. },
$$

where $\beta$ is the effective index and the wave number $k_{0}=\omega / c$. The waveguide is shown in the inset of Fig. 1, where the dielectric constants for the substrate, the film, and the cladding are $\epsilon_{s}, \epsilon_{f}$, and $\epsilon_{c}=\epsilon_{c 0}+\alpha\left|E_{y}\right|^{2}$, respectively. The field solutions in each layer are

$$
\begin{aligned}
& E_{c y}(x)=\left(\frac{2}{\alpha}\right)^{1 / 2} \frac{q}{\cosh \left[k_{0} q\left(x-x_{c}\right)\right]}, \\
& E_{f y}(x)= \begin{cases}A \cos \left[k_{0} \Gamma\left(x-x_{f}\right)\right] & \text { for } \beta^{2}<\epsilon_{f}, \\
A \sinh \left[k_{0} \gamma\left(x-x_{f}\right)\right] & \text { for } \beta^{2}>\epsilon_{f}\end{cases} \\
& E_{s y}(x)=B \exp \left(k_{0} p x\right),
\end{aligned}
$$

where $q=\left(\beta^{2}-\epsilon_{c}\right)^{1 / 2}, \Gamma=\left(\epsilon_{f}-\beta^{2}\right)^{1 / 2}, \gamma=\left(\beta^{2}-\epsilon_{f}\right)^{1 / 2}$, $p=\left(\beta^{2}-\epsilon_{s}\right)^{1 / 2}$, and $A, B, x_{c}$, and $x_{f}$ are constants that can be determined from the boundary conditions and the appropriate effective index. The power of the guided wave per unit length along the $y$ axis is obtained by integrating the Poynting vector over the $x$ axis $^{18}$ :

$$
P=\frac{1}{2} c \epsilon_{0} \beta \int_{-\infty}^{\infty} E_{y}^{2}(x) \mathrm{d} x .
$$

Because the fields depend on the effective index, the power is a function of the effective index. Numerical results are presented below.

The wavelength is $1.3 \mu \mathrm{m}$; the thickness of the film is $5 \mu \mathrm{m}$; the indices are 1.55 and 1.57 for the substrate and the film, respectively, i.e., $\epsilon_{s}=2.4025$ and $\epsilon_{f}=2.4649$; 


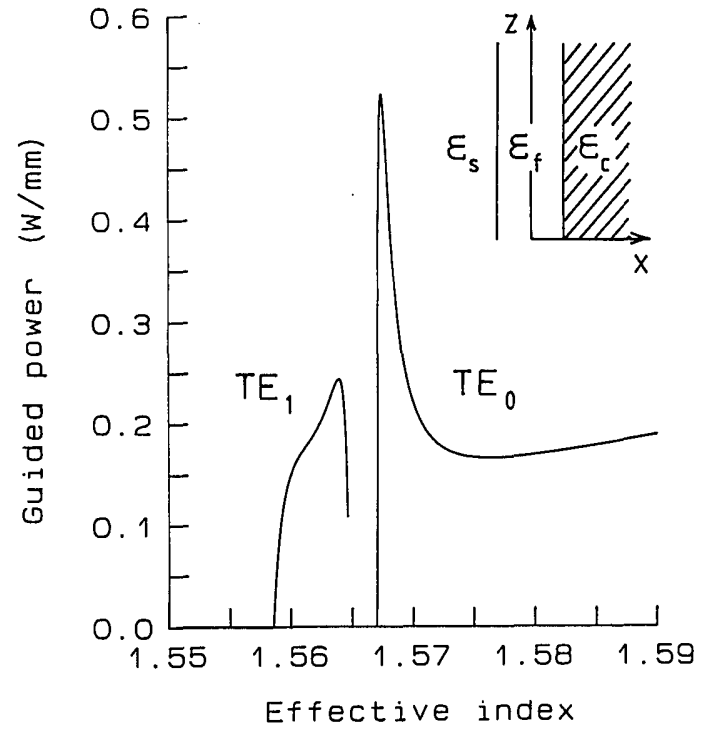

Fig. 1. TE-polarized guided-wave power versus effective index for $\epsilon_{s}=2.4025, \epsilon_{f}=2.4649$, and $\epsilon_{c}=\epsilon_{0}+\alpha|E|^{2}$, where $\epsilon_{0}=2.4025$ and $\alpha=3.3776 \times 10^{-12} \mathrm{~m}^{2} / \mathrm{V}^{2}$. The thickness of the film is $5 \mu \mathrm{m}$, and the wavelength is $1.3 \mu \mathrm{m}$. In the inset the $z$ axis is the propagating axis, the linear substrate is at the left, and the nonlinear cladding is at the right.

the index of the nonlinear cladding is $1.55+n_{2} I$, where $n_{2}=5.3 \times 10^{-10} \mathrm{~m}^{2} /$ W, i.e., $\epsilon_{\mathrm{c}}=\epsilon_{\mathrm{c} 0}+\alpha|E|^{2}$, where $\epsilon_{c 0}=2.4025$ and $\alpha=3.3776 \times 10^{-12} \mathrm{~m}^{2} / \mathrm{V}^{2}$. The nonlinear dispersion curve is depicted in Fig. 1, and the two branches represent the $\mathrm{TE}_{1}$ and $\mathrm{TE}_{0}$ modes. It has been shown that the positively sloped $\mathrm{TE}_{0}$ branches are stable and that this is not strictly true for the $\mathrm{TE}_{1}$ branch. $^{1}$ In this paper losses by absorption and scattering are neglected. If the loss is large, we believe that the soliton emission can be suppressed.

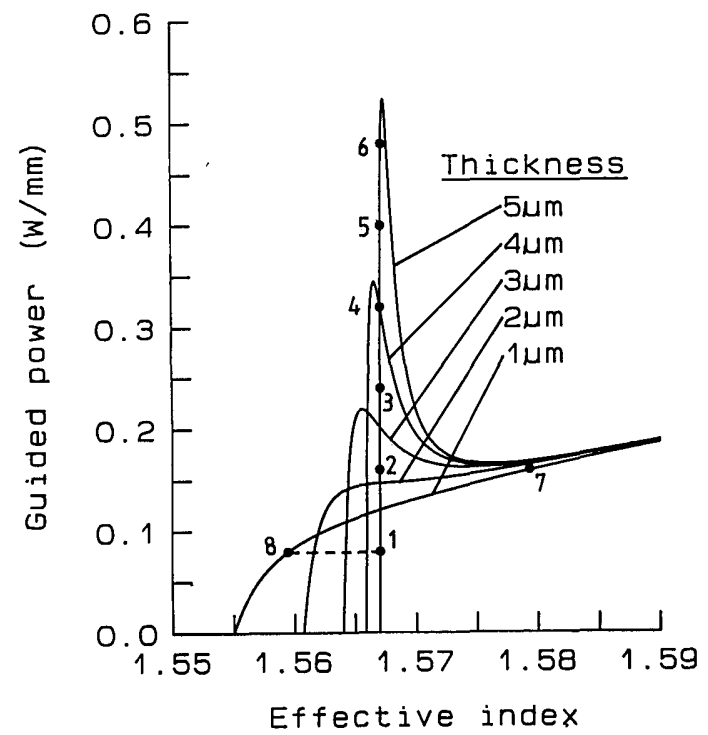

(a)
The nonlinear dispersion curves of the $\mathrm{TE}_{0}$ mode with various film thicknesses are shown in Fig. 2(a). The permittivities of all the layers are the same as in the previous example, and the film thicknesses are $5,4,3,2$, and $1 \mu \mathrm{m}$. The maximum guided power increases as the thickness of the waveguide increases. The curves nearly overlap in the surface-polariton region, which means that the field is approximately the same for different film thicknesses in such modes. For a slowly tapered waveguide it is expected that the wave will evolve along the horizontal line in the figure; i.e., the guided power remains constant and the field shape slowly changes. If the input field is too large for an eigenmode of the thinner waveguide, then it will radiate partially through the spatial soliton. The field evolutions for different input powers are simulated by the beam propagation method; the results and explanations are presented in Section 3.

\section{RESULTS}

Three different situations for wave propagation in the tapered waveguide are simulated. In simulation 1 the wave propagates in the forward direction, with the fundamental mode of the thicker end as the input. In simulation 2 the wave propagates in the backward direction, with the fundamental mode of the thinner end as the input. In simulation 3 the wave propagates in the forward direction, with two modes as the input. In the simulations the beam propagation method is employed; 4096 or 8192 grid points are used in the transverse direction for a 256- or $512-\mu \mathrm{m}$ width, and the propagation step is $0.2 \mu \mathrm{m}$ to ensure that the accumulated numerical errors are negligible.

\section{A. Simulation 1}

Forward propagation with the input being the fundamental mode of the thicker end is simulated. In Fig. 2(a) the

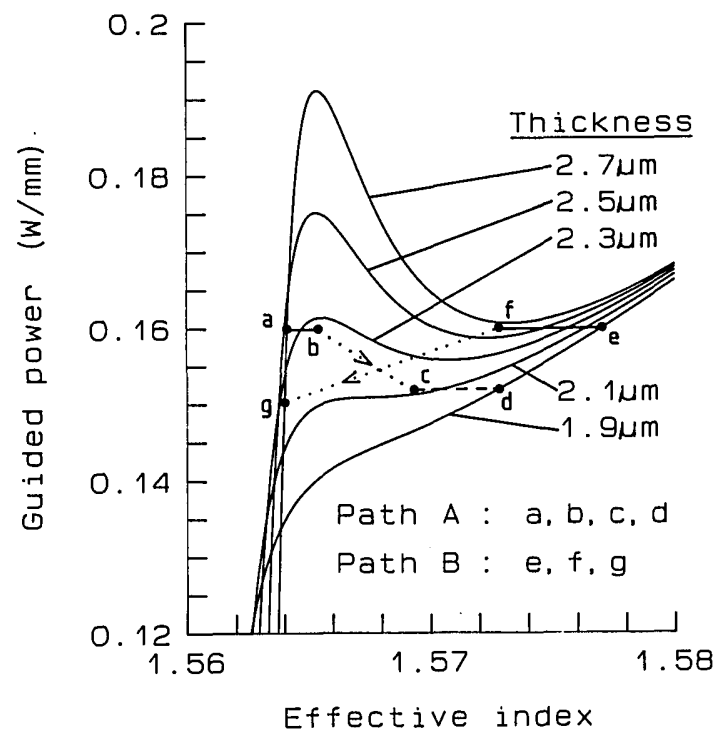

(b)

Fig. 2. (a) $\mathrm{TE}_{0}$ guided-wave power versus effective index for five different film thicknesses: 5, 4, ,3,2, and $1 \mu \mathrm{m}$. Points $1-7$ represent the selected incident fields for the tapered waveguide. The dashed line between points 1 and 8 represents the adiabatic process of the field evolving in the tapered waveguide. (b) Detail of plots of the nonlinear dispersion curves for five different film thicknesses: 2.7, 2.5, 2.3, 2.1, and $1.9 \mu \mathrm{m}$. The jump is the abrupt transition between the guided mode and the surface polariton. Path $A$ is the forward jump, shown in Fig. 3(b), and path B is the backward jump, shown in Fig. 4. The field evolves along the solid horizontal line $a b$ for the adiabatic process in the tapered waveguide before the jump; the dotted lines represent the jumping processes, and the dashed line represents the field evolution after the jump. 

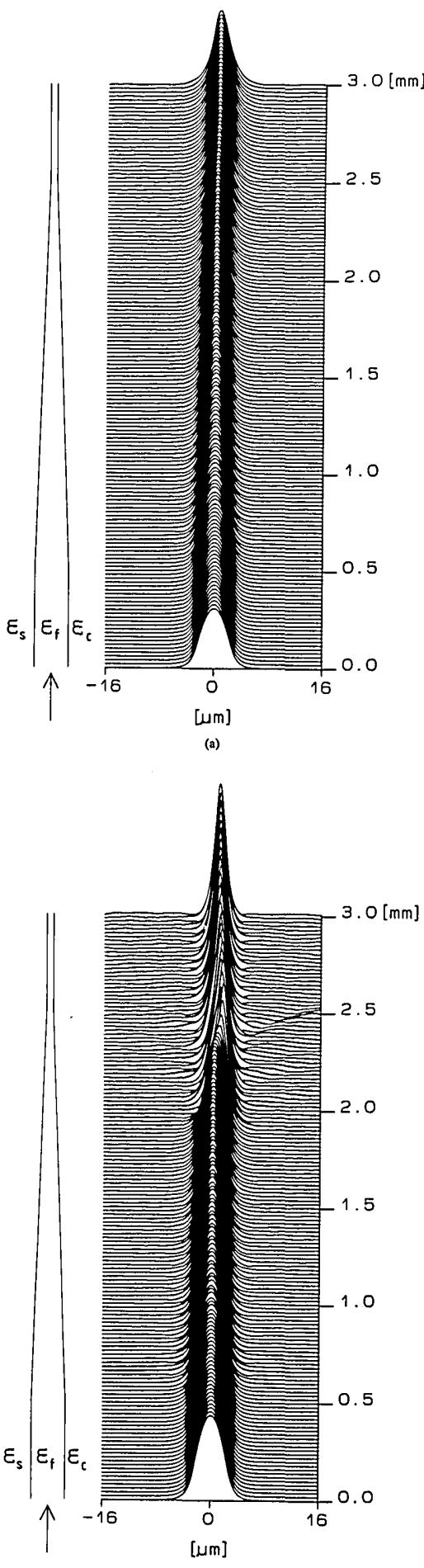

(b)

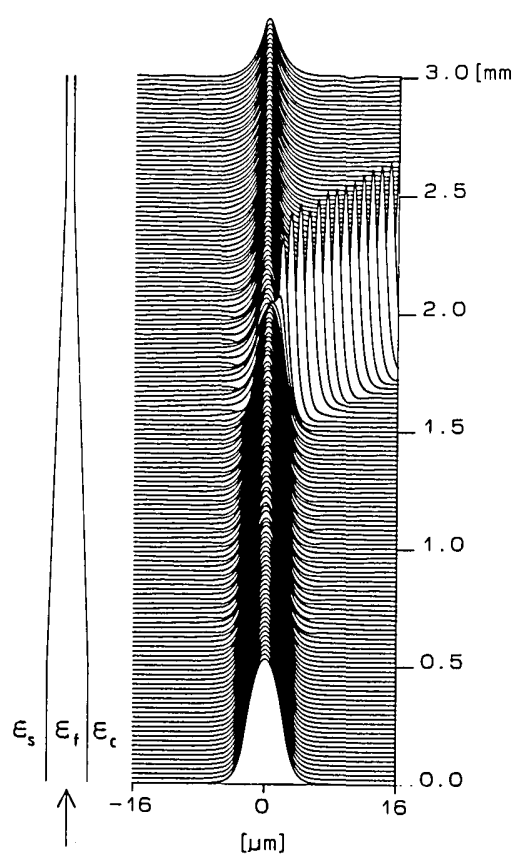

(c)

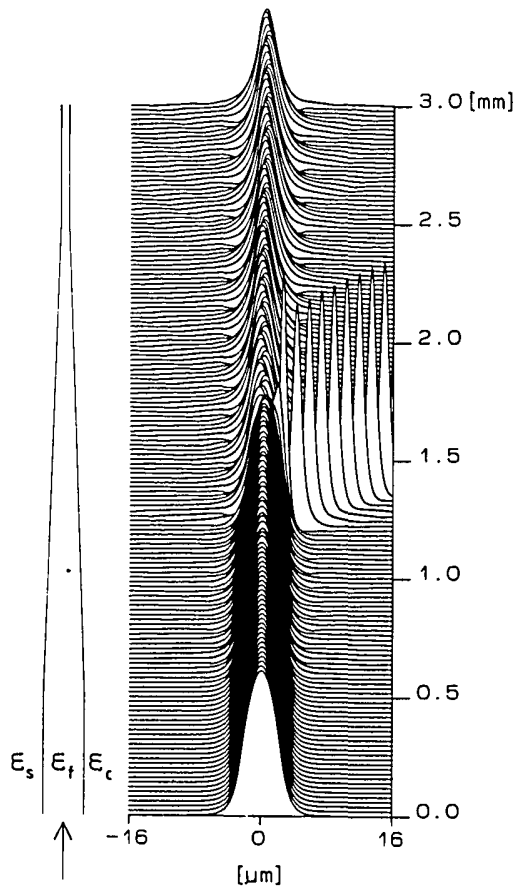

(d)
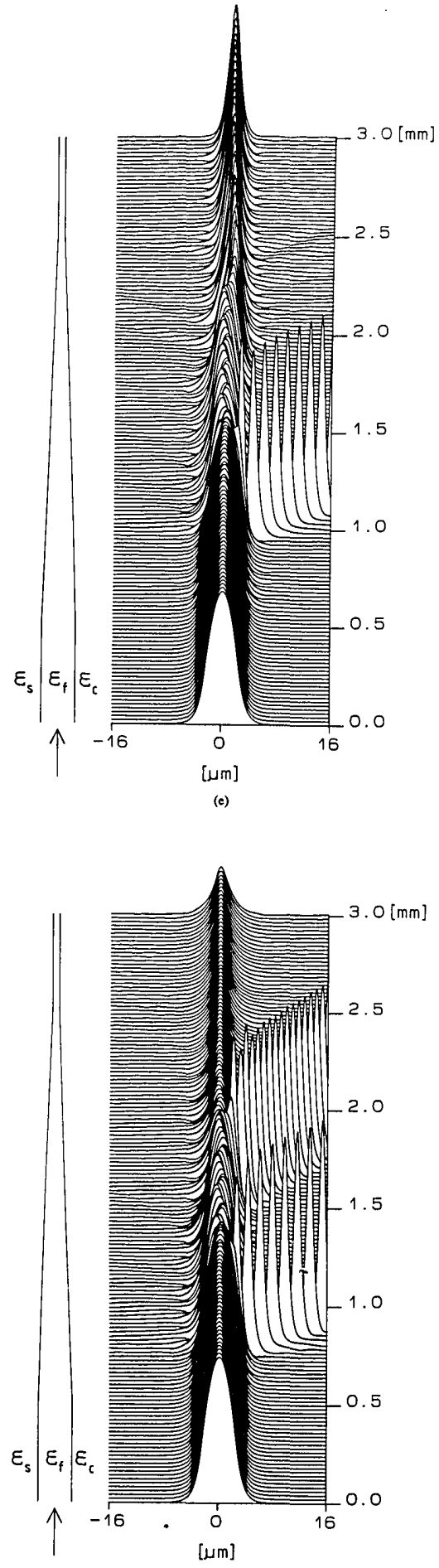

(i)

Fig. 3. Field evolution for forward propagation with various input powers. The tapered waveguide is shown at the left of each panel. The tapered section is $2 \mathrm{~mm}$ long, and the uniform section at each end is $0.5 \mathrm{~mm}$ long. The thickness of the thicker end is $5 \mu \mathrm{m}$ and that of the thinner end is $1 \mu \mathrm{m}$. (a) Input power, $0.08 \mathrm{~W} / \mathrm{mm}$, point 1 of Fig. 2(a). (b) Input power, $0.16 \mathrm{~W} / \mathrm{mm}$, point 2 of Fig. 2(a). (c) Input power, $0.24 \mathrm{~W} / \mathrm{mm}$ point 3 of Fig. 2(a). (d) Input power, $0.32 \mathrm{~W} / \mathrm{mm}$, point 4 of Fig. 2 (a). (e) Input power, $0.4 \mathrm{~W} / \mathrm{mm}$, point 5 of Fig. 2 (a). (f) Input power, $0.48 \mathrm{~W} / \mathrm{mm}$, point 6 of Fig. 2(a).

six powers indicated from the positively sloped $\mathrm{TE}_{0}$ branch of the nonlinear dispersion curve of the thicker end are used as the inputs for forward wave propagation in the tapered waveguide.

For a low input power, e.g., at point 1 in Fig. 2(a), $P_{0}=0.08 \mathrm{~W} / \mathrm{mm}$, the field evolves along the dashed line from point 1 to point 8 , as indicated in Fig. 2(a), and the field profile evolution is shown in Fig. 3(a). As the field propagates to the thinner waveguide, the field profile becomes thinner and the peak of the field moves toward the film-cladding interface. For such low guided power input, the field evolves in an adiabatic process.

For an input power that is twice that in the above case, i.e., point 2 in Fig. 2(a), the evolution of the field is more 
dramatic. Basically, the field still evolves along the horizontal line in Fig. 2(a), but a discontinuity occurs at the point at which the waveguide thickness equals $2.2 \mu \mathrm{m}$ and the maximum guided power is less than the input value. Figure $3(\mathrm{~b})$ shows that the field propagates stably and is compressed by the waveguide, as in the case of low input power, at first. As the thickness of the film decreases further, the field is compressed more and more, and the intensity at the film-cladding interface becomes high enough to radiate energy as a spatial soliton at a propagation distance of $1.9 \mu \mathrm{m}$. The field radiates and is recaptured by the interface immediately. After correlation integral calculation, the recaptured field is shown to be a surface polariton, and the surface polariton swings as it propagates successively along the waveguide. The propagating phenomena described can be illustrated with reference to path A of Fig. 2(b). At first the field evolves adiabatically along the horizontal line through point a to point $b$, shown as a solid line in the figure. As the field propagates successively, there is no corresponding guided mode for the field, and the field tends to radiate. After the field radiates a small amount of power, it becomes a surface polariton at point $c$. Then the field evolves continuously through point $d$ to the corresponding surface polariton of the thinner end. We call this abrupt transition between the guided mode and the surface polariton a jump. The mode considered here is entirely on the positively sloped branches of the dispersion curve. It is called a guided mode when the effective index is less than the film index, and it is called a surface polariton when the effective index is larger than the film index.

For point 3 in Fig. 2(a) the input power $3 P_{0}$ is associated with no corresponding point in the dispersion curve of the waveguide when its film thickness is less than $3.2 \mu \mathrm{m}$; therefore the power is no longer guided, and the field is expected to radiate a spatial soliton in order to carry away the excess power in the waveguide, which is similar to the soliton emission from the uniform nonlinear waveguide. ${ }^{3}$ Because the power radiated is much larger than that of a surface polariton, the field will not be recaptured by the film-cladding interface. The radiated field carries away the most power from the waveguide near a propagation distance of $1.55 \mathrm{~mm}$. In Fig. 3(c) the radiated field is identified as a spatial soliton after correlation integral calculation; the remaining power in the waveguide is small, and only the fundamental mode exists in the waveguide. In the successive tapered process the field evolves adiabatically, as in the case of a low input power.

The guided power is $4 P_{0}$ at point 4 in Fig. 2(a), and the spatial soliton radiation is obvious; the remaining field in the waveguide excites mainly the fundamental mode. The propagating behavior of the remaining field in the waveguide is much like that of two-mode propagation, which is discussed below. The field profile evolution is shown in Fig. 3(d).

The field profile evolution of an input power $5 P_{0}$ is shown in Fig. 3(e). The characteristics of spatial-soliton radiation and the remaining field trapped by the waveguide are similar to those of Fig. 3(d), but the power of the remaining field is higher, and it jumps to the surface polariton in the successive tapered waveguide, as in the case of the $2 P_{0}$ input in Fig. $3(\mathrm{~b})$.

In the incident field at point 6 in Fig. 2(a) two spatial soliton radiations are observed. The remaining field is small after the two successive radiations and evolves adiabatically as shown in Fig. 3(f).

\section{B. Simulation 2}

Backward propagation with the input being the fundamental mode of the thinner end is simulated. The allowed guided power of the guided mode of a thinner waveguide is less than that of a thicker waveguide; the allowed powers are the same for the surface polariton. Thus the thicker waveguide is always able to capture the guided mode that is incident from the thinner waveguide, and spatial soliton radiation is impossible for a guided wave propagating in such a backward-tapered waveguide. Both the guided mode and the surface polariton propagating in the tapered waveguide evolve adiabatically. However, in some specific cases, such as at point 7 in Fig. 2(a), the field evolves with a jump, as shown in Fig. 4. The field jumps from the surface polariton of the thinner waveguide into the guided mode of the thicker end, which is illustrated by path B in Fig. 2(b). The excited guided wave in the successive propagation is similar to the wave propagation of the incident field that is composed of a dominant $\mathrm{TE}_{0}$ wave and a small $\mathrm{TE}_{1}$ wave.

\section{Simulation 3}

Forward propagation with the input being a dominant $\mathrm{TE}_{0}$ wave and a small $\mathrm{TE}_{1}$ wave is simulated. Wave propagation is considered in the forward-tapered direction, because the thinner waveguide supports only a $\mathrm{TE}_{0}$ wave. $\mathrm{A}$ low guided power of the $\mathrm{TE}_{0}$ wave, which will not radiate solitons in the propagation associated with a small $\mathrm{TE}_{1}$ wave, is launched from the thicker end of the tapered waveguide. We would expect the $\mathrm{TE}_{1}$ wave to radiate

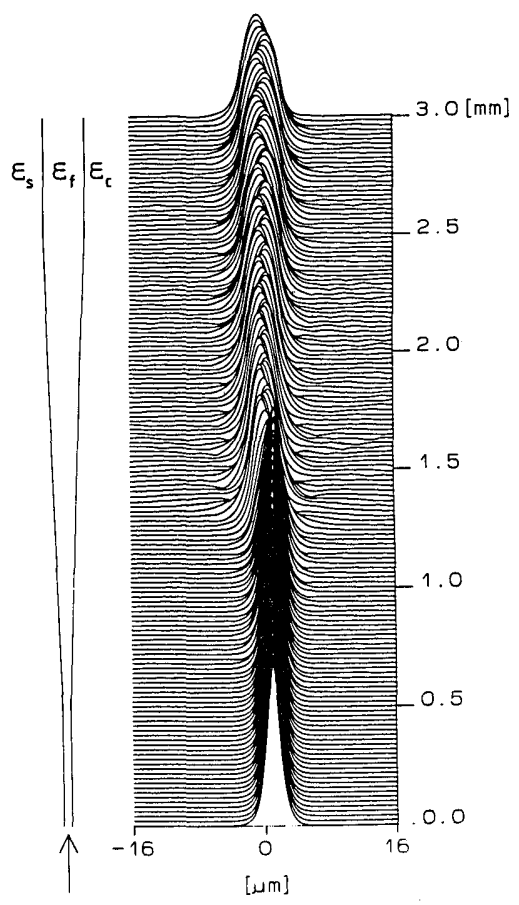

Fig. 4. Profile evolution for backward propagation with an input power of $0.16 \mathrm{~W} / \mathrm{mm}$, point 7 in Fig. 2(a). The tapered waveguide is shown at the left, and its dimension is the same as that in Fig. 3; the linear substrate is to the left and the nonlinear cladding is to the right of the waveguide. 


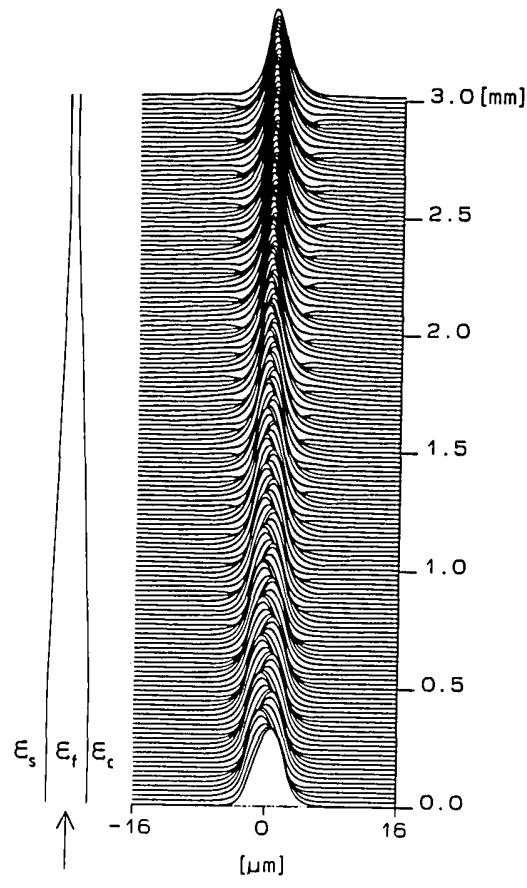

(a)

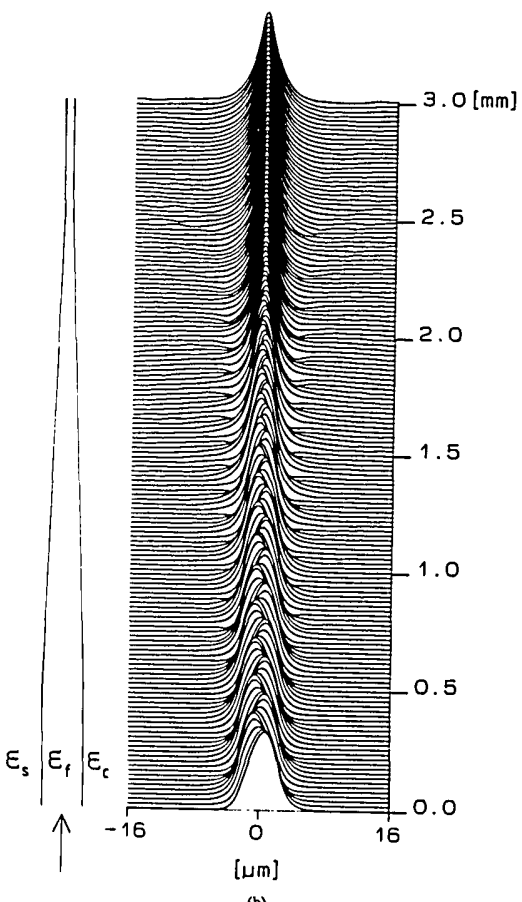

(b)

Fig. 5. (a) Wave propagation in the nonlinear tapered waveguide of the incident field composed of a dominant $\mathrm{TE}_{0}$ wave and a weak $\mathrm{TE}_{1}$ wave. (b) Same as (a) but for the linear case.

beyond cutoff and leave only the $\mathrm{TE}_{0}$ wave in the waveguide. However, on the contrary, the numerical results show that after propagation in the tapered waveguide and in the 20 -mm-long uniform single-mode waveguide the swing of the wave is retained. This result means that the wave propagation of the incident field that is composed of two modes will not approach the fundamental mode that was calculated in Section 2 . The field profile evolution in the nonlinear tapered waveguide is shown in Fig. 5(a), and the linear case is shown in Fig. 5(b) for comparison, where the input powers of the $\mathrm{TE}_{0}$ and $\mathrm{TE}_{1}$ modes are 0.08 and $3.2 \mathrm{~mW} / \mathrm{mm}$, respectively.

\section{APPLICATIONS}

A device with continuous routing of a light beam for photonic switching or spatial scanning is proposed in this section. At the left in Fig. 6(a) the structure of the proposed device is shown schematically. The tapered waveguide, which is the same as that discussed above, terminates with a nonlinear medium. The thicknesses of the thicker and thinner ends of the tapered waveguide are 5 and $1 \mu \mathrm{m}$, respectively. The nonlinear medium at the thinner end is chosen in the same way as the nonlinear cladding of the waveguide; its length here is $0.5 \mathrm{~mm}$.

The characteristics of wave propagation in the tapered waveguide were obtained in Section 3. For a low guided power input the $\mathrm{TE}_{0}$ wave evolves adiabatically, and the power is confined in the waveguide. When a small component of a $\mathrm{TE}_{1}$ wave is present, the field swings even if the thickness of the waveguide is reduced to the singlemode region. We use the field at the end of the tapered waveguide to excite the spatial soliton directly, and the propagation direction of the spatial soliton depends on the phase of the field at the interface between the nonlinear medium and the thinner end of the waveguide. The phase at the interface is determined by the input intensity of the $\mathrm{TE}_{1}$ wave or its phase relative to the $\mathrm{TE}_{0}$ wave. Hence, by changing the input condition, we can control the field propagations in the device. The desired incident $\mathrm{TE}_{0}$ and $\mathrm{TE}_{1}$ waves can be obtained by the converging asymmetric $\mathrm{Y}$ junction. ${ }^{8}$

The input power of the $\mathrm{TE}_{0}$ mode is fixed at $0.12 \mathrm{~W} / \mathrm{mm}$, which is near the maximum allowed power in the tapered waveguide for the adiabatic evolution. For this input power the field evolves adiabatically from the guided mode in the thicker end to the surface-polariton-like mode in the thinner waveguide. Because the shape of the field now is like that of a surface polariton but the effective index is less than the film index, we call the field the surface-polariton-like mode. The surface-polariton-like field in the thinner end excites the spatial soliton with an extremely low radiation loss. The power of the $\mathrm{TE}_{1}$ wave employed here is much less than that of the $\mathrm{TE}_{0}$ wave; thus the spatial soliton is approximately the same in the nonlinear medium for different routes.

\section{A. Power-Controlled Routing}

With reference to Figs. 6(a)-6(c), the routes of the spatial soliton are shown for three input powers of the $\mathrm{TE}_{1}$ wave, which are 0,3 , and $17.4 \mathrm{~mW} / \mathrm{mm}$, corresponding to $0,2.5 \%$, and $14.5 \%$ of the power of the $\mathrm{TE}_{0}$ wave, respectively. For the case of no $\mathrm{TE}_{1}$ wave, the field evolves from the guided mode to the surface-polariton-like mode along the tapered waveguide, and the peak of the field moves toward the film-cladding interface. The phase front of the beam is not parallel to the interface, and the angle of the propagation direction of the spatial soliton is $\sim 0.11^{\circ}$ with respect to the $z$ axis. The angle depends on the tapered angle of the waveguide; it decreases as the tapered angle decreases. The angle of the spatial soliton propagation with respect to the $z$ axis versus the incident power of the $\mathrm{TE}_{1}$ wave is plotted in Fig. 7. The total variation of the angle 

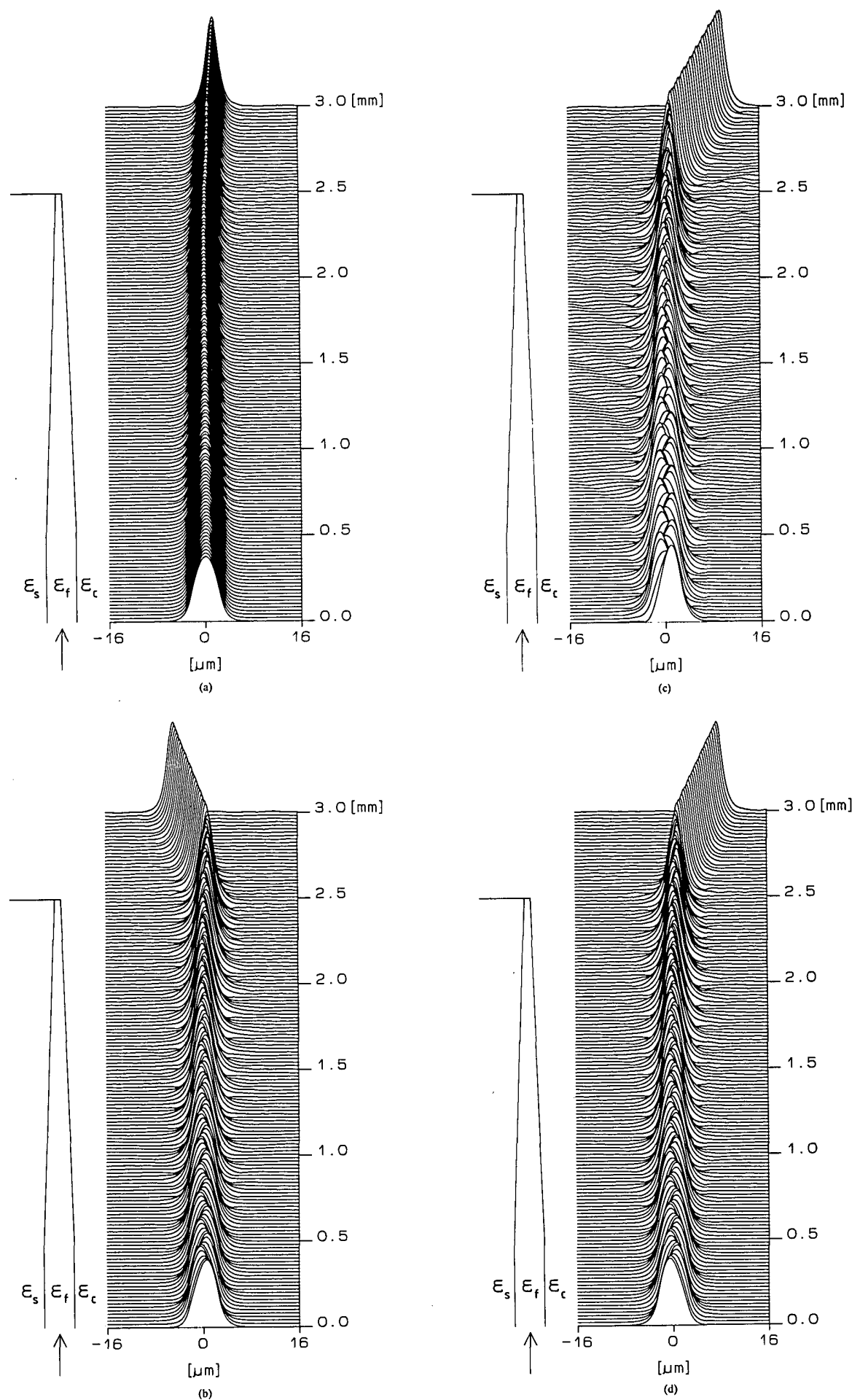

Fig. 6. (a) $\mathrm{TE}_{0}$ wave evolving in the proposed device, which is shown schematically at the left. (b) Same as (a) but with a $\mathrm{TE}_{1}$ wave input of $3 \mathrm{~mW} / \mathrm{mm}$ (i.e., $2.5 \%$ of the power of the $\mathrm{TE}_{0}$ wave). (c) Same as (a) but with a $\mathrm{TE}_{1}$ wave input of $17.4 \mathrm{~mW} / \mathrm{mm}$ (i.e., $14.5 \%$ of the power of the $\mathrm{TE}_{0}$ wave). (d) Same as (b) but with a relative phase difference of $180^{\circ}$ between the $\mathrm{TE}_{0}$ and $\mathrm{TE}_{1}$ waves.

is $\sim 1.6^{\circ}$; for the purpose of switching, the number of allowed channels is proportional to the length of the nonlinear medium. For a beam width of $5 \mu \mathrm{m}$ and a length of $0.5 \mathrm{~mm}$ for the nonlinear medium, the number of allowed channels is 3 .

\section{B. Phase-Controlled Routing}

Routing of the spatial soliton can also be achieved by controlling the phase difference between the $\mathrm{TE}_{0}$ and $\mathrm{TE}_{1}$ waves. The power of the $\mathrm{TE}_{1}$ wave is chosen to be $3 \mathrm{~mW} / \mathrm{mm}, \sim 2.5 \%$ of the power of the $\mathrm{TE}_{0}$ wave, and the power of the $\mathrm{TE}_{0}$ wave is still $0.12 \mathrm{~W} / \mathrm{mm}$. The field profile evolution is shown in Fig. $6(\mathrm{~b})$, where $\mathrm{TE}_{0}$ and $\mathrm{TE}_{1}$ waves are in phase. When the relative phase between two waves is $180^{\circ}$, the field profile evolution is as shown in Fig. 6(d). Figure 8 shows the dependence of the angle of the spatial soliton propagation on the phase difference between the two waves. 


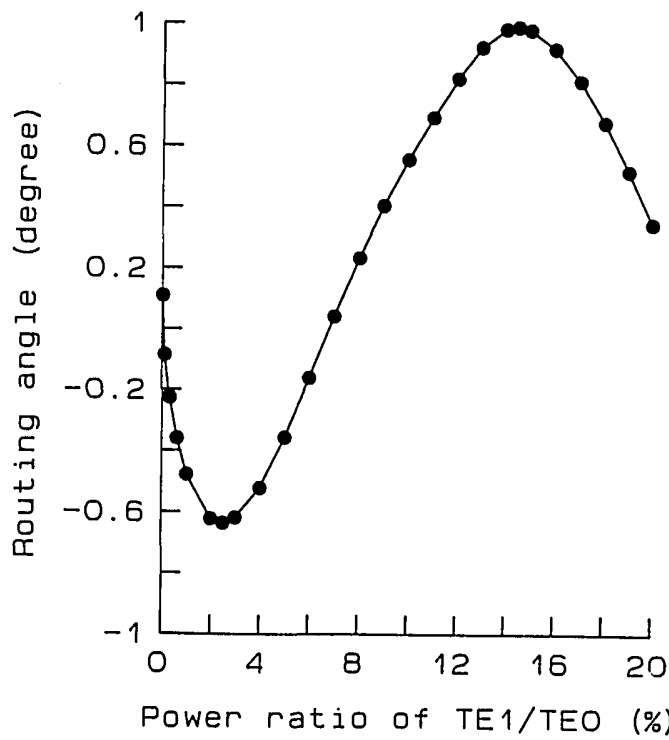

Fig. 7. Dependence of the routing angle on the input power of the $\mathrm{TE}_{1}$ wave.

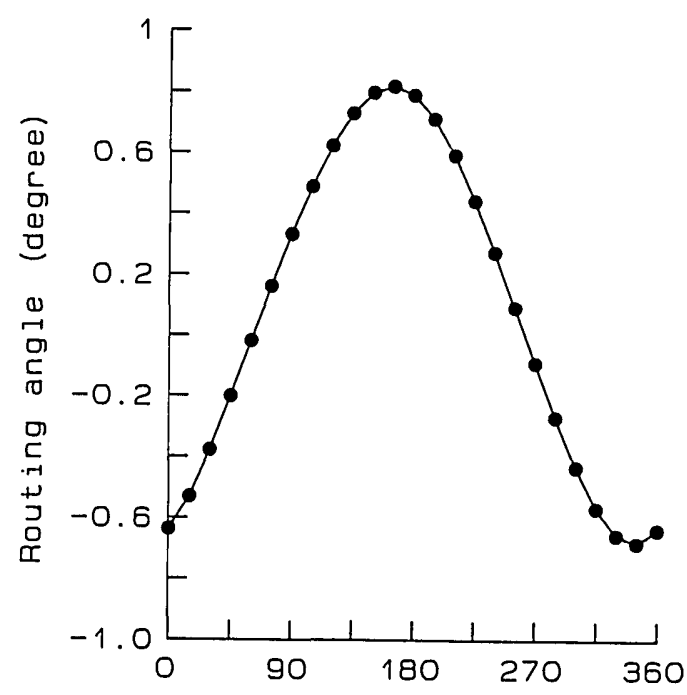

Phase difference (degree)

Fig. 8. Dependence of the routing angle on the relative phase difference between the $\mathrm{TE}_{0}$ and $\mathrm{TE}_{1}$ waves.

\section{CONCLUSIONS}

Field evolution in a tapered nonlinear waveguide has been discussed from the point of view of nonlinear dispersion curves. Since the tapered structure is used in the nonlinear waveguide circuit, the input field of the tapered waveguide is chosen from the stable nonlinear modes. In most cases the available field in the waveguide circuit is the fundamental mode, so the evolution of the $\mathrm{TE}_{0}$ mode is our main interest here.

Both propagation directions of the field in the tapered waveguide are considered. The main difference in the two propagation directions is caused by the different allowable guided powers for different thicknesses of the film. The allowable guided power in the thicker waveguide is larger than that of the thinner waveguide. The propagation's behavior depends on the input powers. When the field is incident in the thicker waveguide with a higher power, the field propagating to the thinner waveguide will radiate the excess power as a spatial soliton. After the radiation, if the remaining part is small it excites the fundamental mode of the waveguide; if the remaining part is large it swings in the propagation. On the other hand, the spatial soliton does not appear for a field propagating from the opposite direction as long as the input field is the guided mode of the thinner waveguide. Jump behavior, which is the abrupt transition between the guided mode and the surface polariton, is observed in both propagation directions. After the jump the excited field is not exactly the eigenmode of the waveguide, and the field swings. For an input power that is less than that required in a jump, the transition between the guided modes and the surface polariton is gradual.

Wave propagation of an incident field that is composed of a strong $\mathrm{TE}_{0}$ wave and a weak $\mathrm{TE}_{1}$ wave in a nonlinear tapered waveguide has been investigated. The swinging effect is observed and lasts for a long distance after the tapered section. We propose a new device for continuously routing the signal by using this property. The spatial soliton is directly excited by the guided field at the end of the tapered waveguide; its propagation direction is determined by the phase of the field in the interface, and the phase depends on the input condition of the two waves. Controlling the input power of the $\mathrm{TE}_{1}$ wave or the phase difference between the $\mathrm{TE}_{0}$ wave and the $\mathrm{TE}_{1}$ wave will allow the signal to be routed in the desired direction.

\section{ACKNOWLEDGMENT}

This research was supported by the National Science Council of the Republic of China under contract NSC-790417-E009-01.

\section{REFERENCES}

1. L. Leine, C. Wächter, U. Langbein, and F. Lederer, "Evolution of nonlinear guided optical fields down a dielectric film with a nonlinear cladding," J. Opt. Soc. Am. B 5, 547-558 (1988).

2. K. Hayata, A. Misawa, and M. Koshiba, "Split-step finiteelement method applied to nonlinear integrated optics," J. Opt. Soc. Am. B 7, 1772-1784 (1990)

3. M. A. Gubbels, E. M. Wright, G. I. Stegeman, C. T. Seaton, and J. V. Moloney, "Numerical study of soliton emission from a nonlinear waveguide," J. Opt. Soc. Am. B 4, 1837-1842 (1987).

4. M. Gubbels, E. M. Wright, G. I. Stegeman, C. T. Seaton, and J. V. Moloney, "Effects of absorption on $\mathrm{TE}_{0}$ nonlinear guided waves," Opt. Commun. 61, 357-362 (1987).

5. L. Leine, C. Wächter, U. Langbein, and F. Lederer, "Propagation phenomena of nonlinear film guided waves in a configuration with material losses: a numerical analysis," Opt. Lett. 12, 747-749 (1987).

6. G. I. Stegeman and E. M. Wright, "All-optical waveguide switching," Opt. Quantum Electron. 22, 95-122 (1990).

7. G. I. Stegeman, E. M. Wright, N. Finlayson, R. Zanoni, and C. T. Seaton, "Third order nonlinear integrated optics," IEEE J. Lightwave Technol. 6, 953-970 (1988).

8. G. I. Stegeman and R. H. Stolen, "Waveguides and fibers for nonlinear optics," J. Opt. Soc. Am. B 6, 652-662 (1989).

9. S. M. Jensen, "The nonlinear coherent coupler," IEEE J. Quantum Electron. QE-18, 1580-1583 (1982).

10. A. Ankiewicz, "Novel effects in nonlinear coupling," Opt. Quantum Electron. 20, 329-337 (1988).

11. L. Thylen, E. M. Wright, G. I. Stegeman, C. T. Seaton, and J. V. Moloney, "Beam-propagation method analysis of a nonlinear directional coupler," Opt. Lett. 11, 739-741 (1986). 
12. D. R. Heatley, E. M. Wright, and G. I. Stegeman, "Soliton coupler," Appl. Phys. Lett. 53, 172-174 (1988).

13. Y. Silberberg and B. G. Sfez, "All-optical phase- and powercontrolled switching in nonlinear waveguide junctions," Opt. Lett. 13, 1132-1134 (1988).

14. J. P. Sabini, N. Finlayson, and G. I. Stegeman, "All-optical switching in nonlinear X-junctions," Appl. Phys. Lett. 55, 1176-1178 (1989).

15. H. Fouckhardt and Y. Silberberg, "All-optical switching in waveguide X-junctions," J. Opt. Soc. Am. B 7, 803-809 (1990).

16. K. Hayata, A. Misawa, and M. Koshiba, "Nonlinear beam propagation in tapered waveguides," Electron. Lett. 25, 661662 (1989).

17. C. T. Seaton, J. D. Valera, R. L. Shoemaker, G. I. Stegeman J. T. Chilwell, and S. D. Smith, "Calculations of nonlinear TE waves guided by thin dielectric films bounded by nonlinear media," IEEE J. Quantum Electron. QE-21, 774-783 (1985).

18. S.-Y. Shin, E. M. Wright, and G. I. Stegeman, "Nonlinear TE waves of coupled waveguides bounded by nonlinear media," IEEE J. Lightwave Technol. 6, 977-983 (1988).

19. M. D. Feit and J. A. Fleck, Jr., "Computation of mode properties in optical fiber waveguides by a propagating beam method," Appl. Opt. 19, 1154-1164 (1980). 